\title{
Relationship Between High Levels of Salivary Cotinine Test and Demographic Characteristics of Pregnant Smokers from Mures County
}

\author{
Ion Mihai Georgescu 1 , Monica Tarcea ${ }^{2}$, Claudiu Marginean ${ }^{3}$, Florina Ruta ${ }^{*}$, Victoria Rus ${ }^{2}$, Remus
} Sipos $^{4}$, Zoltan Abram ${ }^{1}$

\author{
1 Department of Hygiene, University of Medicine and Pharmacy Tirgu Mures \\ 2 Department of Community Nutrition, University of Medicine and Pharmacy Tirgu Mures \\ ${ }^{3}$ Department of Obstetrics and Gynecology I, County Emergency Hospital Tirgu Mures \\ 4 Department of Anatomy, University of Medicine and Pharmacy TirguMures; Mures county General Practitioners Association
}

\begin{abstract}
Objectives: To evaluate the relationship between the frequency of self-declared status regarding smoking in a group of pregnant women from Mures county, Romania and the high levels of Salivary Cotinine (SC) like biomarkers. Material and methods: It was conducted a retrospective study among 230 pregnant women presented for prenatal care at 50 General Practitioners cabinets in Mures county, Romania, in 2015. Data were collected with a validated questionnaire which included age, level of education, socioeconomic status and ethnicity, also the selfreported smoking status. The Salivary Cotinine level was evaluated using NicAlert Saliva test kits. Results: Using salivary test we identified a high prevalence of involuntary exposure to cigarette smoke among both non-smokers and those who quit smoking before pregnancy. Also we registered pregnant women that although declared smoking cessation before pregnancy their salivary Cotinine levels were high, almost like to an active smoker, probably because of second-hand exposure or because they didn't say the truth about their habit. Conclusions: We underline the importance of implementing more efficient community interventions among this vulnerable group in order to reduce the frequency of smoking and sustain quitting.
\end{abstract}

Keywords: smoking, pregnancy, cessation, cotinine test

Received 27 December 2016 / Accepted 23 January 2017

\section{Introduction}

Exposure to cigarette smoke in utero, whether it is direct or indirect via secondhand smoking, can be associated with negative consequences and severe health problems for the newborns, children, and adults [1-5]. Studies revealed that maternal smoking during pregnancy was associated with ectopic pregnancy, placental abruption, prematurity [68], low birth weight babies, and increased risk of having a baby with stunted neuro-development growth and cognitive problems $[9,10,11]$. Pregnant women, who are often aware of the risks of smoking, may be hesitant to disclose their true smoking status during a clinical encounter, limiting opportunities for provider-based counseling and support [12].

The most commonly used biomarker of exposure to tobacco smoke is Cotinine, as a main metabolite of nicotine. The measurement of the Cotinine concentration in various biological fluids is directly proportional to the degree of exposure to nicotine [13]. The determination of Cotinine is recommended for the assessment of active tobacco smoking, monitoring of environmental tobacco smoke (ETS) exposure, and impact evaluation of smoking cessation programs [14].

In a study on pregnant women made in Scotland, there was a $25 \%$ underestimation of smoking using self-reported

* Correspondence to: Florina Ruta

E-mail: florina.ruta@umftgm.ro data that was validated with Cotinine [15]. A similar survey conducted in Sweden revealed that 6\% of self-reported non-smokers were probably smokers and $3 \%$ had Cotinine levels suggestive for secondhand smoking using Cotinine validation [16].

There are scientific proofs that smoke-free environment represents the only strategy for protecting the population from second-hand smoking negative effects [17]. For this matter, many countries have implemented legislations requiring all public places, workplaces and all indoor places to be free of secondhand smoking [18]. In 2004, Ireland was the first country which implemented the smoke-free legislation. Since then, other European and non-European countries followed Ireland; Norway, New Zealand, Italy, Uruguay, England and several provinces in Canada, the USA or Australia [19,20] had implemented the smoke-free law.

In Romania the anti-tobacco law was implemented in June 2002, and stipulate smoking only in specially arranged public spaces and in February 2016 it was modified by banning smoking in all public spaces. A study performed after the adoption of smoke free law in Uruguay showed a measurable decrease of passive smoking exposure in indoor public places and workplaces, the level of exposure was assessed by measuring air nicotine concentrations [21].

The goal of our research was to evaluate the relationship between the frequency of self-declared status regard- 
ing smoking in a group of pregnant women from Mures county, Romania and the high levels of Salivary Cotinine (SC) like biomarkers.

\section{Material And Methods Settings and population}

Population. We conducted a convenience study among pregnant women presented for prenatal care at 50 General Practitioners (GP) cabinets out of 90, in Mures county, Romania. From 324 women which were presented for monitoring purposes to GP's, only a group of 230 pregnant women agreed to fill in a questionnaire regarding smoking status and Cotinine testing, group that formed the final sample of our research.

Measurement: Data were collected based on a validated questionnaire which included age, level of education, socioeconomic status and ethnicity. We also measured selfreported smoking status using the following questions (I have never used cigarettes and I was a regular smoker during pregnancy). Responses were categorized into two groups: smokers and non-smokers.

Salivary Cotinine level was evaluated using NicAlert Saliva test kits. The test principle is based on the use of monoclonal antibodies for Cotinine, and was developed for the identification of smoking status in research studies, on individuals that are monitored for smoking cessation, not for medical diagnosis or therapy. If Cotinine is present in the sample, it will bind to the antibody through binding sites. The number of sites is occupied according to the amount of Cotinine present in a sample. Cotinine, one of the major metabolite of Nicotine, is a suitable candidate as a marker, because it has a relatively long half-life of 10-40 hours and was found to be more sensitive and specific than carbon monoxide in the air.

To achieve regression models we have encoded variables of interest and marked it in the below table 1 as legend that follows: the dependent variable was the amount of Cotinine (the N00, N11, N22 were coded 0 for N33, N44, N55, N66 were coded 1), the independent variables were: Q2 (less than 8 years of school. 8 years of school. Vocational school - coded 1. High school. Graduated. University encoded 0), Q4 (Married. Concubinage - encoded 0. Not married. Divorced. Widow - coded 1), Q5 (Romanian. Hungarian - encoded 0 . Roma people - coded 1 . Than, the level of smoking was evaluated based on), Q7 (I have never used cigarettes. I stopped smoking before I was pregnant I do not smoke. I stopped smoking when I found out I'm pregnant - do not smoke - smoke regularly encoded 0 and for the same number of cigarettes as before I was pregnant. I smoke but I reduced the number of cigarettes after I got pregnant. I increased the number of cigarettes consumed when I found out I was pregnant - coded 1). Q9 (1. coded $0 ; 1.2 .3 .4$ encoded), Q10 (Nobody smokes where I stay. Smokers can smoke only in certain rooms in the house - encoded 0. Smokers can smoke wherever they want coded 1), Q11 (Did not show any change. People smoke anywhere in the house even if they found out they were pregnant - coded 1. No one smokes in the house when I was pregnant - Smoke outside. People smoke in other rooms when they found out I was pregnant - encoded 0), Q18 (I did not smoke cigarettes during the past 30 days. coded 0; Less than one cigarette per day. One cigarette per day. 2-5 cigarettes a day. 6-10 cigarettes per day. Between 11 to 20 cigarettes a day. Between 21 to 30 cigarettes a day. More than 30 cigarettes a day: coded 1), Q19 (I have not smoked a cigarette in the last 30 days - code 0 ; In 5 minutes. In 6-30 min. In 31-60 minutes. After 60 minutes coded 1), and Q25 (Yes. in the next 30 days. Yes. the next 6 months. Yes. but not in the next 6 months - there encoded 0 . I'm not going to give up - coded 1).

\section{Samples Collection/Preparation}

Saliva Testing: Saliva was collected using a funnel, and the collection tube to fill at least one third of its capacity. In the first 4 hours after collection, eight drops of the sample were extracted and deposited on the end of the tape lined Accutest NicAlert test strip, which previously was placed on a flat surface. After transferring saliva from the red zone into the white, the red color must appear in at least one zone (levels 0-6), otherwise, the test results are not valid [25].

Interpretation: Identifying areas of salivary Cotinine labeled bands in the area ranging from $0(0-10 \mathrm{ng} / \mathrm{ml})$ to 10. Any concentration greater than or equal to $10 \mathrm{ng} / \mathrm{ml}$ (zones 1-6) represent a positive result. Salivary Cotinine concentrations and interpretation are as follows: level 0 $(1-10 \mathrm{ng} / \mathrm{ml})$ is a nonsmoker; $11(10-30 \mathrm{ng} / \mathrm{ml})$ corresponds to involuntary smoking; level 22 (30-100ng / $\mathrm{ml}$ ) - confirmed smoker with low tobacco consumption; $33(100-200 \mathrm{ng} / \mathrm{ml})$ is a confirmed smoker with moderate tobacco consumption; and $44(200-500 \mathrm{ng} / \mathrm{ml})-$ is smoker with a high level of tobacco consumption.

The research protocol was approved by the ethics committee at the University of Medicine and Pharmacy TirguMures, as part of a larger study on Building Capacity for Tobacco Research in Romania.

\section{Statistical analysis}

Statistical analysis was performed using the Statistical Package for Social Sciences (SPSS, version 20, Chicago, IL, USA). The association between qualitative variables was assessed using chi-square test or Fisher's exact test. The relationship between the explanatory variables and other Cotinine were evaluated by logistic regression.

The results were presented by odds ratio (OR) and 95\% CIs. For all statistical tests of significance alpha level was set at 0.05 .

\section{Results}

The bivariate analysis of the relationship between Cotinine levels and socio-demographic showed that the smoking status of pregnant women monitored was significantly influenced by the low level of education $(\mathrm{p}=0.0001$. OR: 
5.69 for $95 \%$ CI $0.86-11.25)$ and Roma ethnicity $(\mathrm{p}=$ 0.0001 , OR: 4.9, 95\% CI = 1.90-12.77). No statistically significant data were registered regarding marital status or presence of intervention from a General Practitioner (GP) related to the risk of active or secondhand smoking.

Behavioral parameters data revealed that tobacco use in pregnancy was influenced by smoking status in pre-pregnancy period. Smoking family members also influenced attitudes towards smoking of pregnant women inside the home and those with no changes done during pregnancy about quitting ( $\mathrm{p}=0.0001$, OR: $6.1,95 \% \mathrm{CI}=2.88$ $12.89)$ or ( $\mathrm{p}=0.0001$, OR: $6.1,95 \% \mathrm{CI}=2.67-13.62)$. The lack of change in smoking behavior by direct family members in the presence of pregnant women and inside the house, had a bad impact on tobacco consumption in subjects investigated ( $\mathrm{p}=0.0001$, OR: $2.26,95 \% \mathrm{CI}=$ 1.22-4.20), also the attitude of pregnant women towards smoking in the last 30 days prior to questioning was associated with an increased risk of continued smoking during pregnancy for women who have lit a cigarette at least a day. in the last 30 days ( $\mathrm{p}=0.0001$, OR: $55.5,95 \% \mathrm{CI}=$ 22.22-138.61). The short time between the first cigarette in the morning with the lack of any concerns of cessation, had a significant bad influenced upon smoking status of subjects investigated ( $\mathrm{p}=0.0001$, OR: $56.0,95 \% \mathrm{CI}=$ 22.43-139.79) (see Table I).

In the group of women informed by the GP's about the smoking consequences during pregnancy, was found high levels of Cotinine especially in those with low education, those living in houses where are smokers with no restrictions and those who said they are not planning to quit smoking in the next 30 days.

High levels of Cotinine were found in pregnant smokers, in those with one or more family members who are smokers, those who smoked in the last 30 days, women who smoked within the first hour after awakening, and those that do not think seriously about quitting as well, all subjects from both groups monitored (trained and untrained by the GP's) (see Table II).

Our data showed that an increased level of Cotinine is associated with active smoking (Q7), and with secondhan smoking as well (Q9) (Table III).

An increased Cotinine levels has been highlighted among Roma pregnant women (68.2\%), and in 64.7\% of pregnant women with low education (less than high school) also at $33.6 \%$ of married women.

The level of Cotinine was dependently influenced by the number of smokers in the pregnant household $(\mathrm{p}=$ 0.0004, OR: $5.19,95 \% \mathrm{CI}=2.08-12.96$ ), by the number of cigarettes smoked in the last 30 days $(\mathrm{p}=0.0001$. OR: $73.8,95 \% \mathrm{CI}=19.53-279.31$ ), and how long after awaken $g$ the pregnant light up the first cigarette $(\mathrm{p}=0.0001$, OR: $53.45,95 \% \mathrm{CI}=17.13-79.36$ ).

Our data showed that saliva Cotinine cut-off level of $10 \mathrm{ng} / \mathrm{ml}$ was found to be the optimum cut-off value that differentiates pregnant smokers from non-smokers or secondhand smoking when all testing conditions were possible to fulfill.

Table I. Bivariate analysis of the relationship between Cotinine levels and socio-demographic parameters

\begin{tabular}{|c|c|c|c|c|c|c|}
\hline \multirow{2}{*}{$\begin{array}{l}\text { Socio-demographic parameters } \\
\text { Questions }\end{array}$} & \multirow[b]{2}{*}{ Codes * } & \multicolumn{2}{|c|}{ Cotinine $^{\star \star}$} & \multirow{2}{*}{ OR } & \multirow{2}{*}{ IC } & \multirow{2}{*}{$\mathrm{P}$} \\
\hline & & 1 & 0 & & & \\
\hline \multirow{2}{*}{ Education level } & 1 & $64.7 \%$ & $24.4 \%$ & \multirow{2}{*}{5.69} & \multirow{2}{*}{$2.86-11.25$} & \multirow{2}{*}{0.0001} \\
\hline & 0 & $35.3 \%$ & $75.6 \%$ & & & \\
\hline \multirow{2}{*}{ Marital status } & 1 & $37.5 \%$ & $33.7 \%$ & \multirow{2}{*}{1.18} & \multirow{2}{*}{$0.54-2.57$} & \multirow{2}{*}{0.69} \\
\hline & 0 & $62.5 \%$ & $66.3 \%$ & & & \\
\hline \multirow{2}{*}{ Ethnicity } & 1 & $68.2 \%$ & $30.3 \%$ & \multirow{2}{*}{4.9} & \multirow{2}{*}{$1.90-12.77$} & \multirow{2}{*}{0.0001} \\
\hline & 0 & $31.8 \%$ & $69.7 \%$ & & & \\
\hline \multirow{2}{*}{ Intervention } & 1 & $51.0 \%$ & $45.2 \%$ & \multirow{2}{*}{1.26} & \multirow{2}{*}{$0.51-3.09$} & \multirow{2}{*}{0.64} \\
\hline & 0 & $49.0 \%$ & $54.8 \%$ & & & \\
\hline \multirow{2}{*}{ Time } & Pretest $=1$ & $43.7 \%$ & $69.1 \%$ & \multirow{2}{*}{0.35} & \multirow{2}{*}{$0.19-0.62$} & \multirow{2}{*}{0.0004} \\
\hline & Post-test $=0$ & $56.3 \%$ & $30.9 \%$ & & & \\
\hline \multicolumn{7}{|l|}{ Behavioral parameters } \\
\hline Questions & Codes & & & & & \\
\hline \multirow{2}{*}{ Self-declared smoking status } & 1 & $84.8 \%$ & $10.1 \%$ & \multirow{2}{*}{50.0} & \multirow{2}{*}{ 20.93-119.41 } & \multirow{2}{*}{0.0001} \\
\hline & 0 & $15.2 \%$ & $89.9 \%$ & & & \\
\hline \multirow{2}{*}{ Number of smokers in the family } & 1 & $47.3 \%$ & $12.8 \%$ & 61 & $280-1280$ & $0 \cap 0 \cap 1$ \\
\hline & 0 & $52.7 \%$ & $87.2 \%$ & 6.1 & $2.88-12.89$ & 0.0001 \\
\hline Eynosurn to naccive smoking & 1 & $69.0 \%$ & $27.6 \%$ & 61 & $267-1362$ & $0 \cap 0 \cap 1$ \\
\hline Exposure to passive smoking & 0 & $30.3 \%$ & $72.4 \%$ & 0.1 & $2.07-13.02$ & 0.0001 \\
\hline Avoiding nassive smoking in the family & 1 & $47.8 \%$ & $28.8 \%$ & 226 & $122-420$ & 00001 \\
\hline & 0 & $52.2 \%$ & $71.2 \%$ & 2.20 & $1.22-4.20$ & 0.0001 \\
\hline Smoking status in the last 30 days & 1 & $84.6 \%$ & $9.0 \%$ & 555 & $2222-13861$ & 00001 \\
\hline & 0 & $15.4 \%$ & $91.0 \%$ & 55.5 & & \\
\hline Time duration between -waking and first & 1 & $84.8 \%$ & $9.1 \%$ & 560 & $2243-13979$ & 00001 \\
\hline cigarette in the last 30 days & 0 & $15.2 \%$ & $90.9 \%$ & 56.0 & $22.43-139.19$ & 0.0001 \\
\hline Planning to guit smoking & 1 & $32.3 \%$ & $62.5 \%$ & 0286 & 01100680 & 00001 \\
\hline Planning to quit smokıng & 0 & $67.7 \%$ & $37.5 \%$ & 0.286 & $0.119-0.688$ & 0.0001 \\
\hline
\end{tabular}

${ }^{\star *}$ Bivariate analysis of variable Cotinine, taken binary 0-absent and 1-present. 
Table II. Cotinine levels correlated with socio-demographic parameters, depending on the intervention of GP's or not

\begin{tabular}{|c|c|c|c|c|c|c|c|}
\hline \multirow[b]{2}{*}{ Questions* } & \multirow[b]{2}{*}{ Cotinine $^{\star \star}$} & \multicolumn{3}{|c|}{ Pregnant women with intervention for cessation } & \multicolumn{3}{|c|}{ Pregnant women without intervention for cessation } \\
\hline & & 1 & 0 & $\begin{array}{c}\mathrm{P} \\
\mathrm{OR}(\mathrm{Cl} 195 \%)\end{array}$ & 1 & 0 & $\begin{array}{c}\mathrm{P} \\
\mathrm{OR}(\mathrm{Cl} 195 \%)\end{array}$ \\
\hline \multirow{2}{*}{ Education level } & 1 & 71.4 & 17.6 & \multirow{2}{*}{$\begin{array}{c}0.003 \\
11.6(2.12-64.1)\end{array}$} & 34.6 & 12.0 & \multirow{2}{*}{$\begin{array}{c}0.057 \\
2.88(0.90-16.5)\end{array}$} \\
\hline & 0 & 28.6 & 82.4 & & 65.4 & 88.0 & \\
\hline \multirow{2}{*}{ Marital'status } & 1 & 28.6 & 23.5 & \multirow{2}{*}{$\begin{array}{c}0.75 \\
1.3(0.25-6.52)\end{array}$} & 7.7 & 0.0 & \multirow{2}{*}{$\begin{array}{c}0.15 \\
2.04(1.53-2.71)\end{array}$} \\
\hline & 0 & 71.4 & 76.5 & & 92.3 & 100.0 & \\
\hline \multirow{2}{*}{ Ethnicity } & 1 & 42.9 & 11.8 & \multirow{2}{*}{$\begin{array}{c}0.09 \\
5.62(0.91-34.5)\end{array}$} & 11.5 & 0.0 & \multirow{2}{*}{$\begin{array}{c}0.08 \\
208(1.55-2.80)\end{array}$} \\
\hline & 0 & 57.1 & 88.2 & & 88.5 & 100.0 & \\
\hline \multirow{2}{*}{ Self-declared smoking status } & 1 & 85.7 & 0.0 & \multirow{2}{*}{$\begin{array}{l}0.0001 \\
9.5(2.56-35.2)\end{array}$} & 88.5 & 12.0 & \multirow{2}{*}{$\begin{array}{c}0.0001 \\
56.2(10.23-308.8)\end{array}$} \\
\hline & 0 & 14.3 & 100.0 & & 11.5 & 88.0 & \\
\hline \multirow{2}{*}{ Number of smokers in the family } & 1 & 100.0 & 64.7 & \multirow{2}{*}{$0.44(0.28-0.68)$} & 88.5 & 40.0 & \multirow{2}{*}{$\begin{array}{c}0.0001 \\
11.5(2.71-48.7)\end{array}$} \\
\hline & 0 & 0.0 & 35.3 & & 11.5 & 60.0 & \\
\hline \multirow{2}{*}{ Exposure to passive smoking } & 1 & 50.0 & 0.0 & \multirow{2}{*}{$\begin{array}{c}0.001 \\
3.42(1.83-6.39)\end{array}$} & 15.4 & 12.0 & \multirow{2}{*}{$\begin{array}{c}0.72 \\
1.33(0.26-6.66)\end{array}$} \\
\hline & 0 & 50.0 & 100.0 & & 84.6 & 88.0 & \\
\hline \multirow{2}{*}{ Avoiding passive smoking in the family } & 1 & 35.7 & 17.6 & \multirow{2}{*}{$\begin{array}{c}0.25 \\
2.59(0.49-13.6)\end{array}$} & 44.0 & 35.0 & \multirow{2}{*}{$\begin{array}{c}0.54 \\
1.45(0.43-4.90)\end{array}$} \\
\hline & 0 & 64.3 & 82.4 & & 56.0 & 65.0 & \\
\hline \multirow{2}{*}{ Smoking status in the last 30 days } & 1 & 84.6 & 0.0 & \multirow{2}{*}{$\begin{array}{c}0.001 \\
9.5(2.56-35.2)\end{array}$} & 88.5 & 14.3 & \multirow{2}{*}{$\begin{array}{c}0.001 \\
46.0(8.27-255.6)\end{array}$} \\
\hline & 0 & 15.4 & 100.0 & & 11.5 & 85.7 & \\
\hline \multirow{2}{*}{$\begin{array}{l}\text { Time duration between -waking and first } \\
\text { cigarette in the last } 30 \text { days }\end{array}$} & 1 & 85.7 & 0.0 & \multirow{2}{*}{$\begin{array}{c}0.001 \\
9.5(2.56-35.2)\end{array}$} & 88.5 & 14.3 & \multirow{2}{*}{$\begin{array}{c}0.001 \\
46.0(8.27-255.6)\end{array}$} \\
\hline & 0 & 14.3 & 100.0 & & 11.5 & 85.7 & \\
\hline \multirow{2}{*}{ Planning to quit smoking } & 1 & 0.0 & 43.8 & \multirow{2}{*}{$\begin{array}{c}0.005 \\
2.55(1.53-4.25)\end{array}$} & 40.9 & 57.1 & \multirow{2}{*}{$\begin{array}{c}0.45 \\
0.52(0.09-2.9)\end{array}$} \\
\hline & 0 & 100.0 & 56.2 & & 59.1 & 42.9 & \\
\hline
\end{tabular}

*Legend explained in Methods section.

${ }^{*}$ Multivariate analysis of variable Cotinine, taken binary 0-absent and 1-present.

Table III. Correlations between socio-demographic variables and Salivary Cotinine level

\begin{tabular}{lccc}
\hline Variable & Odds Ratio & $95 \% \mathrm{Cl}$ & $\mathrm{P}$ \\
\hline Intervention & 1.99 & 0.20 to 19.53 & 0.55 \\
Education level & 1.03 & 0.10 to 9.72 & 0.97 \\
Age & 1.09 & 0.89 to 1.34 & 0.37 \\
Marital status & 16.51 & 0.86 to 316.55 & 0.06 \\
Ethnicity & 6.27 & 0.20 to 188.49 & 0.29 \\
Smoking status & 109.12 & 16.76 to 710.38 & $<0.0001$ \\
\hline
\end{tabular}

\section{Discussions}

Starting with estimated rates of daily smoking for $31-37 \%$ of the Romanian adult population and $24 \%$ among students it is critically needed to develop efficient programs for tobacco cessation. In this context, research studies are important to find the best techniques to be used for a sustainable network dedicated to population support for smoking cessation or even better for avoidance of the onset $[13,17]$.

Prolonged exposure to tobacco smoke, especially to cigarettes, has been scientifically proven to have harmful effects on organs, systems and processes in the human body, both for active as for the secondhand smokers. Inhalation of more than 4800 different chemicals by smoking, creates fertility problems and an increased risk of health problems during pregnancy, for mother or fetus [17].

The conditions of appearance and maintenance of this habit are influenced by economic status, education and culture, family background, emotional climate and level of addiction.

After analyzing pregnant self-reports on smoking status, our study showed a high frequency of women who smoked before pregnancy $(30.04 \%)$ of which a high percentage $(43.3 \%)$ continued to smoke during pregnancy, a high frequency even for Romania [22,23,24].

In another similar study conducted in Romania in 2002 on a sample of 286 people, based on sample self reports about smoking status the initial classification was: $50.56 \%$ active smokers, $3.5 \%$ occasional smokers and $55.94 \%$ nonsmokers [26]. After performing the Cotinine test strips the subjects distribution by their smoking status was represented as: $44.06 \%$ active smokers, $39.50 \%$ passive smokers and $16.43 \%$ non-smokers [26]. These results showed that more than a half of the subjects participating in this study self-declared being nonsmokers, but this percent decreased dramatically after the test performing, and higher frequency of positive Cotinine levels was encountered in women than in men $[26,27,28]$.

Regarding the limitations of our study we can mention that there were situations where testing conditions were not always possible to fulfill. In heavy smokers case, their saliva was more thicker than secondhand smokers or nonsmokers women, which did not allowed Cotinine migration into test strip without a thermostatic period. This has not always been possible because not all health facilities within which they carried out the collection were equipped with a thermostat. Thus, there were cases where the Cotinine test showed negative results while the study participants declared themselves as active smokers (6 out of 31 ).

The etiological factor for tobacco addiction is nicotine. The recent smoking cessation approaches tend to focus on this chronic, relapsing dependence on tobacco, even by monitoring components of nicotine in the blood, or sa- 
liva. The success of such approach involves understanding of the chronic nature of tobacco addiction, the monitoring period (not just interventions in the acute stages of manifestation) associated with behavioral educating both for healthcare professionals and also for pacients [20,23].

We believe that the results of this study will enable a shift in starting up community interventions more effective in smoking cessation outcomes, manifest especially among young women who are pregnant or are preparing to be, from different ethnicities and specific socio-demographic and cultural profiles.

\section{Conclusion}

Using salivary Cotinine test we identified a high frequency of involuntary exposure to cigarette smoke among both non-smokers and those who quit smoking before pregnancy. Also we registered pregnant women that although declared smoking cessation before pregnancy their salivary Cotinine levels were high, almost like to an active smoker, probably because of secondhand exposure or because they did not say the truth about their habit. We underline the importance of implementing more efficient community interventions among this vulnerable group in order to reduce the frequency of smoking and sustain quitting.

\section{Acknowledgments}

Research reported that this publication was supported by the Fogarty International Center and the National Cancer Institute of the National Institutes of Health under Award Number R01 TW009280 The content is solely the responsibility of the authors and does not necessarily represent the official views of the National Institutes of Health.

\section{Conflict of interest}

No conflict of interest.

\section{References}

1. Salihu HM, Wilson RE - Epidemiology of prenatal smoking and perinatal outcomes. Early Hum Dev. 2007;83:713-720.

2. Wen CP, Cheng TY, Lin CL, et al - The health benefits of smoking cessation for adult smokers and for pregnant women in Taiwan. Tob Control. 2005;14:156-61.

3. Holbrook BD - The effects of nicotine on human fetal development. Birth Defects Res C Embryo Today. 2016;108:181-92.

4. Balte P, Karmaus W, Roberts G, et al. Relationship between birth weight, maternal smoking during pregnancy and childhood and adolescent lung function: A path analysis. Respir Med. 2016;121:13-20.

5. Rozi S, Butt ZA, Zahid N, at al - Association of tobacco use and other determinants with pregnancy outcomes: a multicentre hospital-based case-control study in Karachi, Pakistan. BMJ Open. 2016;6:111-16.

6. Talati A, Odgerel Z, Wickramaratne PJ, et al - Brain derived neurotrophic factor moderates associations between maternal smoking during pregnancy and offspring behavioral disorders. Psychiatry Res. 2016;245:387-391.
7. Shisler S, Eiden RD, Molnar DS, et al - Effects of fetal tobacco exposure on focused attention in infancy. Infant Behav Dev. 2016;45:1-10.

8. Flanagan CA, Koller KR, Wolfe AW, et al - Fetal Exposure to Carcinogens With Tobacco Use in Pregnancy: Phase 1 MAW Study Findings. Nicotine Tob Res. 2016; 18:2162-2168.

9. England LJ, Aagaard K, Bloch M, al - Developmental toxicity of nicotine: A transdisciplinary synthesis and implications for emerging tobacco products. Neurosci Biobehav Rev. 2016;72:176-189.

10. Hayes C, Kearney M, O'Carroll H, et al - Patterns of Smoking Behaviour in Low - Income Pregnant Women: A Cohort Study of Differential Effects on Infant Birth Weight. Int J Environ Res Public Health. 2016;13:28-33.

11. Leonardi-Bee J, Smyth A, Britton J, et al - Environmental tobacco smoke and fetal health: systematic review and meta-analysis. Arch Dis Child Fetal Neonatal. 2008;93:351-361.

12. Okoli C - A Comparison of Survey Measures and Biomarkers of Secondhand Tobacco Smoke Exposure among Nonsmokers. Public Health Nurs. 2016;33(1):82-9.

13. Fagan $\mathrm{P}$, Moolchan ET, Pokhrel P, et al - Addictive Carcinogens Workgroup: Biomarkers of tobacco smoke exposure in racial/ethnic groups at high risk for lung cancer. Am J Public Health. 2015;105:123745.

14. SRNT Subcommittee on Biochemical Verification, "Biochemical verification of tobacco use and cessation," Nicotine and Tobacco Research, 2012;4(2):149-159.

15. Shipton D, Tappin DM, Vadiveloo T, et al - Reliability of self reported smoking status by pregnant women for estimating smoking prevalence: a retrospective, cross sectional study. BMJ. 2009;339:b4347.

16. Lindqvist $\mathrm{R}$, Lendahls $\mathrm{L}$, Tollbom $\mathrm{O}$, et al - Smoking during pregnancy: comparison of self-reports and Cotinine levels in 496 women. Acta Obstet Gynecol Scand. 2002; 81:240-244.

17. World No Tobacco Day: smoke-free inside: create and enjoy $100 \%$ smoke-free environments. WHO Library Cataloguing-in-Publication Data. 2007.

18. Fernández E, Fu M, Pascual JA, et al - Impact of the Spanish smoking law on exposure to secondhand smoke and respiratory health in hospitality workers: a cohort study. PLoS One. 2009;4:e4244.

19. Akhtar PC, Currie DB, Currie CE, et al - Changes in child exposure to environmental tobacco smoke (CHETS) study after implementation of smoke-free legislation in Scotland: national cross sectional survey. $\mathrm{Br}$ Med J. 2007;335:545

20. Franchini M, Caruso C, Perico A, et al - Assessment of foetal exposure to cigarette smoke after recent implementations of smoke-free policy in Italy. Acta Paediatr. 2008; 97:546-550.

21. Blanco-Marquizo A, Goja B, Peruga A, et al - Reduction of secondhand tobacco smoke in public places following national smoke-free legislation in Uruguay. Tob Control. 2010;19(3):231-234.

22. Arora CP, Kacerovsky M, Zinner B, et al - Disparities and relative risk ratio of preterm birth in six Central and Eastern European centers. Croat Med J. 2015;56(2):119-27.

23. Meghea CI, Rus IA, Cherecheș RM, et al - Maternal smoking during pregnancy and birth outcomes in a sample of Romanian women. Cent Eur J Public Health. 2014;22(3):153-8.

24. Trofor A, Man MA, Miron R - Smoking during pregnancy-a challenge to practitioners. Pneumologia. 2009;58(4):247-249.

25. Accutest ${ }^{\circledR}$ NicAlert ${ }^{\top M}$ Saliva - Jant Pharmacal. Never Place NicAlert Strip in Mouth, http://www.accutest.net/products/pdf/ds47ny150.pdf (Accesed November 2016).

26. Nuca C, Amariei C, Badea V, et al - Salivary Cotinine - biomarker of tobacco consumption in the assessment of passive smoking prevalence. Farmacia. 2012; 60(5):662-674.

27. Shipton $D$, Tappin $D$, Vadiveloo $T$, et al - Reliability of self reported smoking status by pregnant women for estimating smoking prevalence: a retrospective, cross sectional study. BMJ. 2009;339:b4347.

28. Polanska K, Krol A, Kaluzny P, et al - Estimation of Saliva Cotinine Cut-Off Points for Active and Passive Smoking during Pregnancy - Polish Mother and Child Cohort. Int J Environ Res Public Health, 2016;13(12):1216. 